\title{
Analytical Method Development and Validation for Assay of Rufinamide Drug
}

\author{
JITENDER SINGH ${ }^{*}$, SONIA SANGWAN ${ }^{1}$, PARUL GROVER ${ }^{1}$, \\ LOVEKESH MEHTA ${ }^{1}$, DEEPIKA KIRAN ${ }^{1}$ and ANJU GOYAL ${ }^{2}$ \\ ${ }^{1}$ Lord Shiva College of Pharmacy, Sirsa, Haryana, India \\ ${ }^{2}$ Chitkara College of Pharmacy, Chandigarh-Patiala National Highway, \\ Rajpura, Patiala, Punjab, India \\ E-mail: saggujittu@yahoo.com
}

\begin{abstract}
A simple, rapid, sensitive, cost effective and reproducible reverse phase high performance liquid chromatographic (RP-HPLC) method was developed and validated for the stability testing of rufinamide. The proposed RP-HPLC method was developed on phenome-nex Luna ${ }^{\mathrm{R}} \mathrm{C}-185 \mu \mathrm{m}, 250 \mathrm{~mm}$ $\times 4.6 \mathrm{~mm}$ id. Column (at ambient temperature) and mobile phase consisting of phosphate buffer: acetonitrile (60:40) was delivered at a flow rate $1.0 \mathrm{ml} /$ $\min$. The analyte was detected by using UV detector at the wavelength of 293 $\mathrm{nm}$. The method was found to be linear over the concentration range of 50$150 \mathrm{\mu gml}^{-1}\left(\mathrm{r}^{2}=0.999\right)$. 30. The retention time of rufinamide was $4.717 \mathrm{~min}$.
\end{abstract}

Keywords: RP-HPLC, Rufinamide, API, Method Validation

\section{INTRODUCTION}

tability testing forms an important part in the process of drug product development. Active pharmaceutical ingredient (API) is the important part of drug formulation and drug degrades with time so there is a need to develop methods which can detect degradation as well as degraded products (Chafez, L., 1971 and Sethi, P.D., 2001). The purpose of stability testing is to provide evidence on how the quality of drug substance varies with time under the influence of variety of environmental factors such as temperature, humidity and light which enables recommendation of storage conditions, retest periods and shelf life.

Journal of Pharmaceutical Technology, Research and Management Volume 1, No. 2, November 2013 pp. 191-203

\subsection{Drug Profile (Rufinamide)}

Description: (1-[(2, 6-difluorophenyl)methyl]triazole-4-carboxamide)

Rufinamide is an anti-epileptic is an structural analogue of MK801, carbamazepine and valproic acid recent analogue to gabapentin.

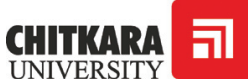

(C)2013 by Chitkara University. All Rights Reserved. 
Singh, J.

Sangwan, S.

Grover, P.

Mehta, L.

Kiran, D.

Goyal, A.<smiles>NC(=O)c1cn(Cc2c(F)cccc2F)nn1</smiles>

Figure: Rufinamide structure

\section{Physiochemical properties Rufinamide}

$\begin{array}{ll}\text { Parameter } & \text { Values } \\ \text { Molecular Weight } & 238.19 \\ \text { Physical state } & \text { White crystalline po } \\ \text { Melting point } & 237-240^{\circ} \mathrm{C} \\ \text { Solubility in Water } & \text { Very low Soluble in } \\ \text { Stability } & \text { Stable under ordinary } \\ \text { Assay } & 98.5 \% \\ \text { Residue on Ignition } & 0.15 \% \text { max } \\ \text { Loss on Drying } & 9.5-12.5 \% \text { max } \\ \text { Optical Rotation } & -0.82^{\circ} \\ \text { Heavy Metal } & \geq 20 \text { ppm } \\ \text { Category } & \text { Anti-epileptic Drug } \\ \text { State } & \text { Solid } \\ \text { Half Life } & 6-10 \text { hours }\end{array}$

\subsection{Method Development}

Number of methods are available to carryout stability indicating assay e.g. gas chromatography and nuclear magnetic resonance (NMR) (Chatwal, G., Anand, S.K., 2004 and Riley, M., Rosanke, T.W., 1996). High performance liquid chromatography (HPLC) is supposed to be the most efficient because it utilizes columns packed with very smaller particles and higher flow rate, which provides improved resolution, speed and sensitivity. Additional advantage of HPLC is that stability indicating assay of drugs can be carried out effectively in a short time (Boubakar, B.B., Etienne, R., et al., 2001; Hong, D., Shah, M., 2000 and Kar, A., 2005). 


\section{EXPERIMENTAL WORK}

\subsection{Materials}

\subsubsection{Instruments}

The HPLC system consisted of HPLC 10AT-VP (Shimdadzu corporation ltd., Kyoto, Japan), manual injector port with $20 \mu \mathrm{L}$ fixed loop (Rheodyme USA), UV detector SPD-20A (Shimadzu Corporation Ltd., Kyoto, Japan) and LC20 AT (Prominenceseries) pumps were used. Separation was carried out on phenomenax C18, column $(250 \mathrm{~mm} \times 4.6 \mathrm{~mm}, 5 \mu \mathrm{m})$ Japan. Detector output was quantified on Spinchrom CFR chromatography software. Mettler-Toledo International Inc. Greifensee (Switzerland) microbalance was used for the purpose of weighing, and spinix vortex was used for the mixing. Samples were injected into HPLC system using Hamilton micro syringe.

\subsection{Chemicals and Reagents}

A gift sample of pure rufinamide was supplied from Varda Biotech pvt. Limited, Mumbai. Acetonitrile, water, methanol, potassium dihydrogen phosphate and triethylamine were procured from M/S Qualigens Fine Chemicals, Mumbai.

\subsection{Methods}

\subsubsection{Selection of wavelength}

$10 \mathrm{ppm}$ of drug solution was prepared by dissolving $100 \mathrm{mg}$ of drug in small amount of methanol and volume was adjusted to $100 \mathrm{~mL}$; after that $1 \mathrm{ml}$ of above solution was diluted to $100 \mathrm{~mL}$ with methanol to get final concentration of $10 \mathrm{ppm}$. The solution was scanned in the U.V range of $200 \mathrm{~nm}$ to $400 \mathrm{~nm}$ (Green, J.M., 1996).

\subsubsection{Preparation of Mobile Phase}

For the analysis of rufinamide the aqueous system selected was phosphate buffer. Dissolved $6.08 \mathrm{Gm}$ of potassium dihydrogen phosphate in sufficient water to produce $1000 \mathrm{~mL}$. $\mathrm{pH}$ was adjusted with glacial acetic acid using $\mathrm{pH}$ meter. After that buffer was mixed and sonicated with organic solvent i.e. acetonitrile in the ratio of $60: 40$ (Taylor and Francis, 2007).

\subsubsection{Preparation of Calibration Curve}

Stock solution $\left(50 \mu \mathrm{gmL}^{-1}\right)$ of rufinamide was prepared by dissolving in mobile phase. The stock solution of rufinamide was further diluted with mobile phase
Analytical Method Development and Validation for Assay of Rufinamide Drug 
Singh, J.

Sangwan, S.

Grover, P.

Mehta, L.

Kiran, D.

Goyal, A. to give the series of standard dilution for preparation of calibration curve (Taylor and Francis., 2007). The different concentration of sample solution $\left(12.5,25,50,62.5,75,100 \mathrm{\mu gml}^{-1}\right)$ was injected in the concentration range of $50 \%-150 \%$ of drug substanceand the Calibration Curve results are presented in Table 1 and Figure 1.

Table 1: Linearity data for Rufinamide

\begin{tabular}{lccccc}
\hline S.No. & $\begin{array}{c}\text { Concentration/ } \\
\boldsymbol{\mu g m l}^{-1}\end{array}$ & Inj-1/Area & Inj-2/Area & Inj-3 Area & $\begin{array}{c}\text { Average area } \\
\text { count/mV*sec }\end{array}$ \\
\hline 1 & 12.5 & 336 & 338 & 340 & 338 \\
2 & 25 & 668 & 662 & 674 & 668 \\
3 & 50 & 1368 & 1333 & 1340 & 1337 \\
4 & 62.5 & 1680 & 1696 & 1703 & 1693 \\
5 & 75 & 1998 & 2009 & 2008 & 2005 \\
6 & 100 & 2675 & 2688 & 2650 & 2671 \\
& & & & Slope & 26.71 \\
& & & & Intercept & 4.71 \\
& & & & $\mathrm{R}^{2}$ & 0.999 \\
\hline
\end{tabular}

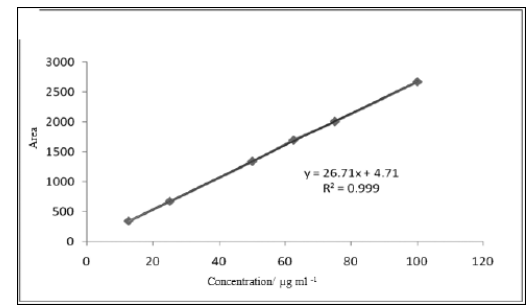

Figure 1. Linear calibration curve of rufinamide (Area vs Concentration $/ \mu \mathrm{g} / \mathrm{ml}^{-1}$ )

\subsubsection{Preparation of standard solution and test solution}

Standard and test stock solutions $(50 \mathrm{ppm})$ of rufinamide were prepared using $100 \mathrm{mg}$ of standard and test sample of rufinamide. Drugs was dissolved in 25 $\mathrm{mL}$ sof methanol and sonicated for $10 \mathrm{~min}$. Then the volume was made up to $100 \mathrm{~mL}$ with diluent. After that $5 \mathrm{~mL}$ of above solution was diluted up to 100 $\mathrm{mL}$ with methanol. 


\subsubsection{Chromatographic Conditions}

Analytical Method

\section{Optimisation of chromatographic conditions}

The mobile phase for the proposed method (Phosphate buffer $\mathrm{pH} 4$ : Acetonitrile 60 : 40) was filtered through $0.45 \mu \mathrm{m}$ membrane filter. It was degassed with a sonicator for $15 \mathrm{~min}$ and pumped from the reservoir to the column (Phenomenex C-18, $250 \mathrm{~mm} \times 4.6 \mathrm{~mm}, 5 \mu \mathrm{m}$ ) at aflow rate of $1 \mathrm{~mL}$

\section{Standard}

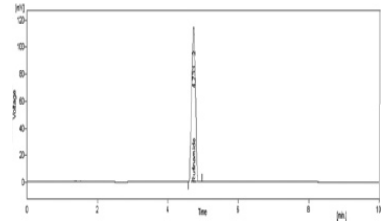

Figure 2

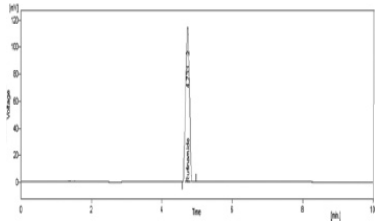

Figure 3 Development and Validation for Assay of Rufinamide Drug

Sample Chromatogram

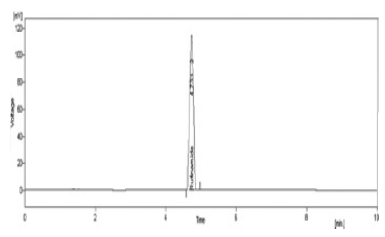

Figure 4

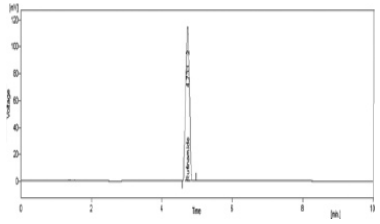

Figure 6

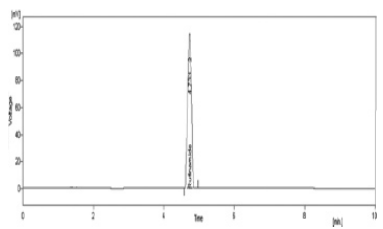

Figure 8

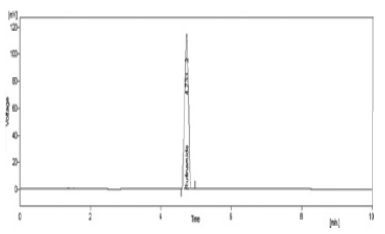

Figure 5

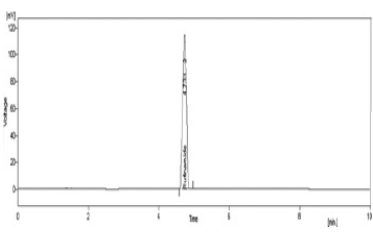

Figure 7

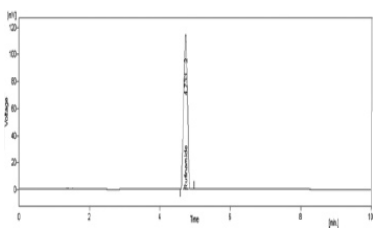

Figure 9 
Singh, J.

Sangwan, S.

Grover, P.

Mehta, L.

Kiran, D.

Goyal, A.

$\min ^{-1}$. The run time was set at $10 \mathrm{~min}$. Prior to injection of the drug solutions the column was equilibrated for at least $1 \mathrm{~h}$ with mobile phase flowing through the system. The analyte was monitored at $225 \mathrm{~nm}$ and data acquired was stored and analyzed with spinchrom CFR chromatography software.

\subsection{Method Validation Studies}

Selectivity of the method was assessed on the basis of elution of Rufinamide using the above mentioned chromatographic conditions. The linearity, precision, accuracy, limit of detection, limit of quantitation and robustness has been validated for the determination of Rufinamide.

\subsubsection{System precision}

Six replicate injections of standard solution were given and mean of all of these values gives rise to the RSD value obtained. According to USP \%RSD (Relative Standard Deviation) should not be more than 2\% (Riley, M., Rosanke, T.W., 1996).

\subsubsection{Method Precision}

Method precision or Intra-assay precision data were obtained by repeatedly analyzing, in onelaboratory on one day, aliquots of homogeneous sample, each of which were independently prepared according to method procedure (Sethi, P.D., 2001 and Kar, A., 2005).

\subsubsection{Linearity}

Linearity of method is determined in the range 50-150 $\mu \mathrm{g} \mathrm{mL} \mathrm{m}^{-1}(50 \%-150 \%)$. According to International Conference on Harmonisation (I.C.H) guidelines correlation coefficient should be less than 0.999 (ICH, 1996).

\subsubsection{Ruggedness}

This analysis was repeated with different column on different day with different analyst and different system and \%RSD value was determined.

\subsection{Stability in Analytical Solution}

A drug solution of $50 \mathrm{ppm}$ was prepared and kept at room temperature i.e. $25^{\circ} \mathrm{C}$ for $24 \mathrm{hrs}$. After that drug solution was analyzed and it was found to be stable at room temperature (Chafez, L., 1971).

\subsubsection{Degradation studies of Rufinamide}

The drug was allowed to degrade in acidic, basic, oxidative and thermal conditions (Chafez, L., 1971). 


\section{RESULTS AND DISCUSSION}

Analytical Method

Development and Validation

\subsection{System precision}

The system precision was analyzed by six replicate injections each of standard solutions of rufinamide $(50 \mathrm{ppm})$ into the HPLC system and the results are presented in Table 2. Percentage RSD for system precision was found to be $0.75 \%$.

Table 2: Different trials carried out for developing the current HPLC

\begin{tabular}{|c|c|c|c|c|c|c|}
\hline $\begin{array}{l}\text { S. } \\
\text { No }\end{array}$ & Column Used & Mobile Phase & Mode & $\begin{array}{l}\text { Injection } \\
\text { Vol. }\end{array}$ & Observation & Result \\
\hline 6.1 & $\begin{array}{c}\text { Phenomenex } \\
\operatorname{luna}^{\mathrm{R}} \mathrm{C} 18(4.6 \times \\
250) \mathrm{mm}, 5 \mu \mathrm{m}\end{array}$ & $\begin{array}{c}\text { Ammonium acetate } \\
(\mathrm{pH}-6.7): \text { Can }(60 \\
: 40)\end{array}$ & Isocratic & $20 \mu \mathrm{l}$ & $\begin{array}{c}\text { Peak shape was } \\
\text { not good }\end{array}$ & $\begin{array}{l}\text { Method } \\
\text { rejected }\end{array}$ \\
\hline 6.2 & $\begin{array}{c}\text { Phenomenex } \\
\operatorname{luna}^{\mathrm{R}} \mathrm{C} 18(4.6 \times \\
250) \mathrm{mm}, 5 \mu \mathrm{m}\end{array}$ & $\begin{array}{c}\text { Ammonium acetate } \\
(\mathrm{pH}-6.7): \text { Can }(55 \\
: 45)\end{array}$ & Isocratic & $20 \mu \mathrm{l}$ & $\begin{array}{l}\text { Peak shape } \\
\text { was not ok } \\
\text { (Fronting) }\end{array}$ & $\begin{array}{l}\text { Method } \\
\text { rejected }\end{array}$ \\
\hline 6.3 & $\begin{array}{c}\text { Phenomenex } \\
\text { luna }{ }^{\mathrm{R}} \mathrm{C} 18(4.6 \times \\
250) \mathrm{mm}, 5 \mu \mathrm{m}\end{array}$ & $\begin{array}{c}\text { Ammonium acetate } \\
(\mathrm{pH}-2.5): \text { ACN : } \\
\text { Methanol } \\
(60: 30: 10)\end{array}$ & Isocratic & $20 \mu \mathrm{l}$ & $\begin{array}{l}\text { Peak width was } \\
\text { more (peak } \\
\text { shape was not } \\
\text { good) }\end{array}$ & $\begin{array}{l}\text { Method } \\
\text { rejected }\end{array}$ \\
\hline 6.4 & $\begin{array}{c}\text { Phenomenex } \\
\text { luna }{ }^{\mathrm{R}} \mathrm{C} 18(4.6 \times \\
250) \mathrm{mm}, 5 \mu \mathrm{m}\end{array}$ & $\begin{array}{c}\text { Triethylamine } \\
(\mathrm{pH}-3): \mathrm{ACN} \\
(70: 30)\end{array}$ & Isocratic & $20 \mu \mathrm{l}$ & $\begin{array}{l}\text { Extra peak as } \\
\text { interferences } \\
\text { were present }\end{array}$ & $\begin{array}{l}\text { Method } \\
\text { rejected }\end{array}$ \\
\hline 6.5 & $\begin{array}{c}\text { Phenomenex } \\
\text { luna }{ }^{\mathrm{R}} \mathrm{C} 18(4.6 \times \\
250) \mathrm{mm}, 5 \mu \mathrm{m}\end{array}$ & $\begin{array}{c}\text { Triethylamine } \\
(\mathrm{pH}-3): \mathrm{ACN} \\
(50: 50)\end{array}$ & Isocratic & $20 \mu \mathrm{l}$ & $\begin{array}{c}\text { Tailing was } \\
\text { more and } \\
\text { absorbance was } \\
\text { less }\end{array}$ & $\begin{array}{l}\text { Method } \\
\text { rejected }\end{array}$ \\
\hline 6.6 & $\begin{array}{c}\text { Phenomenex } \\
\text { luna }{ }^{\mathrm{R}} \mathrm{C} 18(4.6 \times \\
250) \mathrm{mm}, 5 \mu \mathrm{m}\end{array}$ & $\begin{array}{c}\text { Triethylamine } \\
\text { (pH-3) : Methanol } \\
(80: 20)\end{array}$ & Isocratic & $20 \mu \mathrm{l}$ & $\begin{array}{l}\text { More run time, } \\
\text { peak shape was } \\
\text { not good }\end{array}$ & $\begin{array}{l}\text { Method } \\
\text { rejected }\end{array}$ \\
\hline 6.7 & $\begin{array}{c}\text { Phenomenex } \\
\operatorname{luna}^{\mathrm{R}} \mathrm{C} 18(4.6 \times \\
250) \mathrm{mm}, 5 \mu \mathrm{m}\end{array}$ & $\begin{array}{c}\text { Triethylamine } \\
\text { (pH-4) : Methanol } \\
(75: 25)\end{array}$ & Isocratic & $20 \mu \mathrm{l}$ & $\begin{array}{c}\text { peak shape was } \\
\text { not good }\end{array}$ & $\begin{array}{l}\text { Method } \\
\text { rejected }\end{array}$ \\
\hline 6.8 & $\begin{array}{c}\text { Phenomenex } \\
\text { luna }{ }^{\mathrm{R}} \mathrm{C} 18(4.6 \times \\
250) \mathrm{mm}, 5 \mu \mathrm{m}\end{array}$ & $\begin{array}{c}\text { Buffer }\left(\mathrm{Na}_{2} \mathrm{HPO}_{4}+\right. \\
\left.\mathrm{KH}_{2} \mathrm{PO}_{4}\right)(\mathrm{pH}-4): \\
\mathrm{ACN}(50: 50)\end{array}$ & Isocratic & $20 \mu \mathrm{l}$ & $\begin{array}{c}\text { Peak shape was } \\
\text { not good }\end{array}$ & $\begin{array}{l}\text { Method } \\
\text { rejected }\end{array}$ \\
\hline 6.9 & $\begin{array}{c}\text { Phenomenex } \\
\text { luna }{ }^{\mathrm{R}} \mathrm{C} 18(4.6 \times \\
250) \mathrm{mm}, 5 \mu \mathrm{m}\end{array}$ & $\begin{array}{c}\text { Buffer }\left(\mathrm{Na}_{2} \mathrm{HPO}_{4}+\right. \\
\left.\mathrm{KH}_{2} \mathrm{PO}_{4}\right)(\mathrm{pH}-4): \\
\mathrm{ACN}(60: 40)\end{array}$ & Isocratic & $20 \mu \mathrm{l}$ & $\begin{array}{l}\text { Extra peaks as } \\
\text { interference } \\
\text { were present }\end{array}$ & $\begin{array}{l}\text { Method } \\
\text { rejected }\end{array}$ \\
\hline 6.10 & $\begin{array}{c}\text { Phenomenex } \\
\text { luna }{ }^{\mathrm{R}} \mathrm{C} 18(4.6 \times \\
250) \mathrm{mm}, 5 \mu \mathrm{m}\end{array}$ & $\begin{array}{c}\text { Buffer }\left(\mathrm{Na}_{2} \mathrm{HPO}_{4}+\right. \\
\left.\mathrm{KH}_{2} \mathrm{PO}_{4}\right)\left(\mathrm{pH}_{4}\right): \\
\mathrm{ACN}(60: 40)\end{array}$ & Isocratic & $20 \mu \mathrm{l}$ & $\begin{array}{c}\text { Both peak shape } \\
\text { and absorbance } \\
\text { were good }\end{array}$ & $\begin{array}{l}\text { Method } \\
\text { accepted }\end{array}$ \\
\hline
\end{tabular}


Singh, J.

Sangwan, S.

Grover, P.

Mehta, L.

Kiran, D.

Goyal, A.

\subsection{Method Precision}

Two injections of standard solutions of rufinamide $(50 \mathrm{ppm})$ were injected to check the system suitability. Then six sample rufinamide each batch were prepared separately andinjected in duplicate. Results are presented in Table 3, then \%RSD for method precision was found to be $0.77 \%$.

Table 3: Six replicate injection of the stock solution (50ppm)

\begin{tabular}{ll}
\hline Injection & Area counts/mV*sec \\
\hline 1 & 1324 \\
2 & 1342 \\
3 & 1350 \\
4 & 1348 \\
5 & 1332 \\
6 & 1333 \\
Mean & 1338 \\
SD & 10 \\
$\%$ RSD & 0.75 \\
\hline
\end{tabular}

\subsection{Linearity}

Linearity was determined by injecting six replicate injections of standard solutions of rufinamide $(50 \mathrm{ppm})$ to check the system suitability. Then, the different concentration of sample solution was injected in duplicate in the concentration range of 50\%-150\% of drug substance, and the results are presented in Table 3. The correlation coefficient was found to be 0.999 from six replicate injections.

\subsection{Ruggedness}

Analysis was carried out with different analyst, using different column and different day and the results are presented in Table 4 . The \%RSD for ruggedness was found to be $0.44 \%$.

\subsection{Robustness}

Robustness is the capacity of a method to remain unaffected by small deliberate variations in method parameters. Such as change in flow rate $( \pm$ $10 \%), \mathrm{pH}( \pm 0.2$ units $)$ and organic content $( \pm 2 \%)$. The results are presented in Table 6.9- Table 6.17. along with system suitability parameters of normal methodology. 
Table 4. Method precision for calibration data Rufinamide

\begin{tabular}{|c|c|c|c|c|c|}
\hline $\begin{array}{l}\text { Type of } \\
\text { Sample }\end{array}$ & Weight/mg & $\begin{array}{c}\text { Inj-1 } \\
\text { (Area) }\end{array}$ & $\begin{array}{c}\text { Inj-2 } \\
\text { (Area) }\end{array}$ & $\begin{array}{c}\text { Mean area } \\
\text { counts/mV*sec }\end{array}$ & $\begin{array}{c}\text { Assay } / \% \\
w_{w} / w^{-1}\end{array}$ \\
\hline Standard & 50.16 & 1324 & 1342 & 1333 & \\
\hline Sample 1 & 50.16 & 1338 & 1348 & 1343 & 99.82 \\
\hline Sample 2 & 50.02 & 1340 & 1336 & 1338 & 99.72 \\
\hline Sample 3 & 50.85 & 1346 & 1339 & 1343 & 98.46 \\
\hline Sample 4 & 50.16 & 1340 & 1364 & 1352 & 100.49 \\
\hline Sample 5 & 50.12 & 1350 & 1346 & 1348 & 100.27 \\
\hline \multirow[t]{4}{*}{ Sample 6} & 49.98 & 1348 & 1346 & 1347 & 100.48 \\
\hline & & & & $\begin{array}{l}\text { Mean Assay/ } \\
\% \mathrm{w} / \mathrm{w}^{-1}\end{array}$ & 99.87 \\
\hline & & & & SD & 0.765 \\
\hline & & & & $\% \mathrm{RSD}$ & 0.77 \\
\hline
\end{tabular}

\section{Standard}

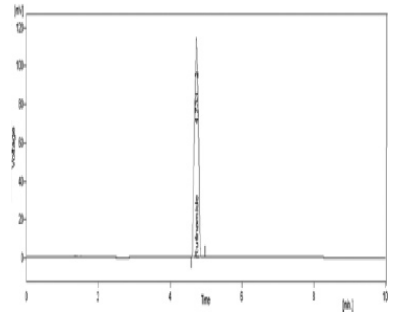

Figure 10
Analytical Method Development and Validation for Assay of Rufinamide Drug 
Singh, J.

Sangwan, S.

Grover, P.

Mehta, L.

Kiran, D.

Goyal, A.

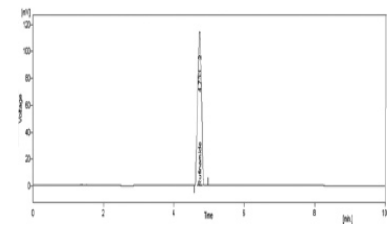

Figure 14

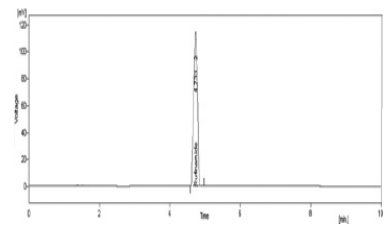

Figure 16

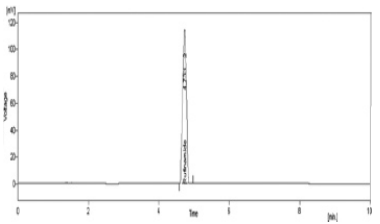

Figure 15

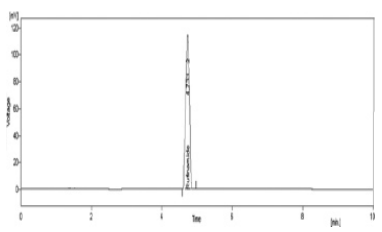

Figure 17

Table 5. Rufinamide Ruggedness Assay

\begin{tabular}{|c|c|c|c|c|c|}
\hline $\begin{array}{l}\text { Type of } \\
\text { Sample }\end{array}$ & Weight/mg & $\begin{array}{l}\text { Inj-1 } \\
\text { (Area) }\end{array}$ & $\begin{array}{l}\text { Inj-2 } \\
\text { (Area) }\end{array}$ & $\begin{array}{c}\text { Mean area } \\
\text { counts } / V^{*} \text { sec }\end{array}$ & $\begin{array}{c}\text { Assay } / \% \\
\text { ww }^{-1}\end{array}$ \\
\hline Standard & 50.26 & 1358 & 1372 & 1365 & 99.87 \\
\hline Sample 1 & 50.06 & 1359 & 1368 & 1368 & 99.98 \\
\hline Sample 2 & 50.02 & 1358 & 1362 & 1362 & 99.77 \\
\hline Sample 3 & 50.04 & 1363 & 1367 & 1367 & 99.14 \\
\hline Sample 4 & 50.03 & 1348 & 1359 & 1359 & 99.31 \\
\hline Sample 5 & 50.06 & 1352 & 1366 & 1366 & 99.61 \\
\hline \multirow[t]{4}{*}{ Sample 6} & 50.05 & 1364 & 1353 & 1353 & 99.63 \\
\hline & & & & $\begin{array}{c}\text { Mean Assay/ } \\
\% w^{-1}\end{array}$ & 99.63 \\
\hline & & & & SD & 0.473 \\
\hline & & & & $\%$ RSD & 0.44 \\
\hline
\end{tabular}

\subsection{Stability in Analytical Solution}

The sample was found to be stable at $25^{\circ} \mathrm{C}$ for $24 \mathrm{hrs}$ and the overall \%RSD was found to be 0.45 and the result are presented in Table 7. 
Table 6: Robustness parameter

\begin{tabular}{lcc}
\hline Parameter & \% RSD & \%Assay \\
\hline Increased Wavelength (+5) & 0.74 & 98.88 \\
Decreased Wavelength (-5) & 0.64 & 98.86 \\
Increased pH (- 0.2) & 0.69 & 99.38 \\
Decreased pH (+ 0.2) & $0 . .69$ & 99.65 \\
Increased Flow rate (+0.1ml) & 0.84 & 98.79 \\
Decreased Flow rate (-0.1ml) & 0.77 & 99.59 \\
Organic content increased & 0.66 & 99.58 \\
Organic content decreased & 0.71 & 99.19 \\
\hline
\end{tabular}

Analytical Method Development and Validation for Assay of Rufinamide Drug

Table 7: Stability data for Rufinamide (at $25^{\circ} \mathrm{C}$ ):

\begin{tabular}{lcccccc}
\hline S.NO. & Time & $\begin{array}{c}\text { Interval/ } \\
\text { hrs }\end{array}$ & $\begin{array}{c}\text { Interval } \\
/ \text { min }\end{array}$ & $\begin{array}{c}\text { Area } \\
\text { counts/ } \\
\boldsymbol{\mu v * s e c}\end{array}$ & $\begin{array}{c}\text { Cumulative } \\
\text { RSD(\%) }\end{array}$ & Assay \\
\hline 1 & $6 / 8 / 201014: 00$ & & & 1338 & 0.11 & 99.55 \\
2 & $6 / 8 / 201014: 30$ & $0: 30: 00$ & 30 & 1340 & 0.15 & 99.70 \\
3 & $6 / 8 / 20103: 00: 00$ & $1: 00: 00$ & 60 & 1336 & 0.46 & 99.41 \\
& PM & & & & & \\
4 & $6 / 8 / 201017: 00$ & $3: 00: 00$ & 180 & 1350 & 0.61 & 100.45 \\
5 & $6 / 8 / 201020: 00$ & $6: 00: 00$ & 360 & 1355 & 0.69 & 100.82 \\
6 & $6 / 9 / 20108: 00$ & $18: 00: 00$ & 1080 & 1330 & 0.69 & 98.96 \\
7 & $6 / 9 / 201014: 00$ & $24: 00: 00$ & 1440 & 1332 & 0.45 & 99.11 \\
& & & & Mean\% & 0.45 & 99.71 \\
& & & & RSD & & \\
\hline
\end{tabular}

\subsection{Degradation studies of Rufinamide}

The drug was allowed to degrade in acidic, basic, oxidative and thermal conditions and results are presented in Table 8 . There was no co eluting peaks (Figure 18).

\section{RESULTS}

Figure 2 shows typical chromatogram of Rufinamide. System stability tests were carried out on freshly prepared standard stock solutions of Rufinamide at $25^{\circ} \mathrm{C}$ (Table 5). The calibration curve was linear in the range of 50-150 $\mu \mathrm{g} / \mathrm{ml}$ for rufinamide. The degradation studies of Rufinamide are shown in Table 6 . 
Singh, J.

Sangwan, S.

Grover, $\mathrm{P}$.

Mehta, L.

Kiran, D.

Goyal, A.

Table 8: Degradation studies of Rufinamide:

\begin{tabular}{lc}
\hline Degradation studies & \% degradation \\
\hline Alkaline degradation $(0.05 \mathrm{~N} \mathrm{NaOH}, 1 \mathrm{~h})$ & 3 \\
Alkaline degradation $(0.01 \mathrm{~N} \mathrm{NaOH}, 1 \mathrm{~h})$ & 7 \\
Alkaline degradation $(1 \mathrm{~N} \mathrm{NaOH}, 1 \mathrm{~h})$ & 13 \\
Alkaline degradation $(2 \mathrm{~N} \mathrm{NaOH}, 1 \mathrm{~h})$ & 15 \\
Alkaline degradation $(5 \mathrm{~N} \mathrm{NaOH}, 1 \mathrm{~h})$ & 25 \\
Acidic degradation $(0.1 \mathrm{~N} \mathrm{HCl})$ & 16 \\
Oxidative degradation $\left(1 \% \mathrm{H}_{2} \mathrm{O}_{2}\right)$ & 11 \\
Oxidative degradation $\left(3 \% \mathrm{H}_{2} \mathrm{O}_{2}\right)$ & 20 \\
Thermal degradation $\left(\right.$ solid sample, $\left.100{ }^{\circ} \mathrm{C}, 24 \mathrm{~h}\right)$ & 0 \\
Thermal degradation $\left(\right.$ solid sample, $\left.100{ }^{\circ} \mathrm{C}, 24 \mathrm{~h}\right)$ & 0 \\
\hline
\end{tabular}

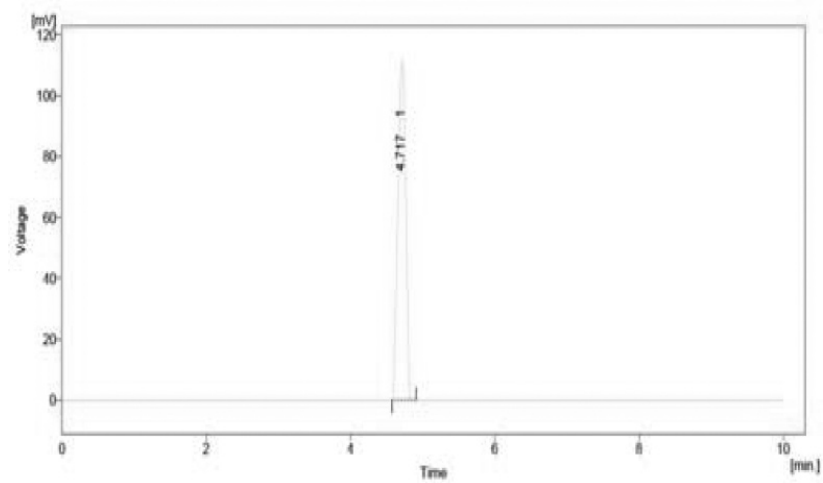

Figure 18. Chromatogram of Rufinamide. (The peak retention time of Rufinamide was $4.717 \mathrm{~min}$ )

\section{CONCLUSION}

HPLC method was successfully developed and validated for determination of rufinamide. The total run time was $10 \mathrm{~min}$. Method validation results have proved the method to be selective, precise, accurate, robust and stability indicating. Thus, the developed stability indicating assay method can be successfully applied for routine analysis of rufinamide.

\section{REFERENCES}

Beckett, A.H and Stanlake, J.B., (2002). Practical Pharmaceutical Chemistry. $4^{\text {th }}$ Edn, Part 2, CBS Publishers and Distributors. 
Boubakar, B.B., Etienne, R., Ducint, D., Quentinc, S., (2001). An HPLC method was developed and validated for the estimation of Moxifloxacin in growth media. Journal of chromatography. B: Biomedical sciences and Applications. 754(1), 107-112.

Chafez, L., (1971). Stability-indicating assay methods for drugs and their dosage forms.Journal of Pharmaceutical Sciences. 60, 335. http://dx.doi.org/10.1002/jps.2600600302

Chatwal, G., Anand, S.K., (2004).Instrumental methods of chemical analyses. $5^{\text {th }}$ ed, Himalayas publishing house, India.

Green, J.M., (1996). A Practical Guide to Analytical Method Validation. Analytical Chemistry. 68, 305A-309A. http://dx.doi.org/10.1021/ac961912f

Hong, D. and Shah, M., (2000). Development and Validation of HPLC Stability Indicating Assays in Drug Stability Principles \& Practice. Marcel. Decker, New York, pp 338.

ICH, (1996). Validation of Analytical Procedure. International Conference on Harmonization. IFPMA, Geneva, 739-49.

Kar, A., (2005). Pharmaceutical Drug Analysis. Second edition, New Age International (P) Ltd. Publishers, New Delhi, 453-476.

Riley, M., Rosanke, T.W., (1996). Development and Validation of Analytical Method. Biddle Ltd., 46-70.

Sethi, P.D., (2001). HPLC Quantitative Analysis of Pharmaceutical Formulations. 1st ed, New Delhi: CBS Publishers \& Distributors, 3.

Taylor and Francis, (2007). Developed Differential pulse polarographic method for the determination of Moxifloxacin in Pharmaceutics,serum and urine. Analytical letters. 40(3), 529-546.

http://dx.doi.org/10.1080/00032710600964817 